\title{
Begleitverletzungen bei distaler Radiusfraktur
}

\author{
Johannes Frank, Arne Kelm, Ingo Marzi
}

\section{Zusammenfassung}

Verletzungen des distalen Radius mit Verkürzung und Verkippung führen nicht selten zu einer Mitverletzung von stabilisierenden Bandstrukturen - insbesondere des Discus triangularis (TFC) und des skapholunären Bandes. Neben diesen begleitenden Weichteilverletzungen dürfen Frakturen der Handwurzelknochen nicht übersehen werden. Gerade der jüngere Patient, dessen Frakturursache meist auf einer hohen Krafteinwirkung beruht, ist da gefährdet, und es muss auf eine Skaphoidfraktur oder eine, als Maximalvariante, Fraktur in Kombination mit Luxation geachtet werden. Eine ausbleibende bzw. verspätete Versorgung dieser Begleitverletzungen führt $\mathrm{zu}$ funktionellen Defiziten. Diese Verletzungen erfordern häufig eine differenzierte klinische, radiologische oder arthroskopische Diagnostik. Neben einer möglichst anatomischen Wiederherstellung des distalen Radius müssen daher die Verletzungen des distalen Radioulnargelenks (DRUG), des Discus triangularis (TFC) und der wichtigen Band- bzw. Knochenstrukturen beachtet werden. Das funktionelle Ergebnis ergibt sich daher aus der anatomischen Wiederherstellung des distalen Radius einschließlich seiner unmittelbaren Begleitstrukturen.

\section{Concomitant Injuries in Distal Radius Fractures}

Fractures of the distal radius with shortening and dislocation lead in some cases to a concomitant lesion of the stabilising ligaments - especially of the triangular fibrocartilage (TFC) and the scapholunate ligaments. Besides these soft-tissue injuries, fractures of the carpal bones must be addressed. Especially young patients are at risk due to the fact that such lesions result from a high impact force. Therefore there is a need to search for carpal fractures or, as worst possible case, a combination with joint luxation. Missing or delayed care of such concomitant lesions will end in functional deficiency. These injuries often require differentiated clinical, radiological or arthroscopic diagnostics. In addition to a nearly anatomic restoration of the distal radius, injuries of the DRUJ, the TFC, the major ligamentous structures and fractures of the carpal bones need to be addressed. The functional outcome is related to the anatomic restoration of the distal radius including its direct neighbouring structures.

\section{Einleitung}

Der komplexe anatomische Aufbau des Handgelenks und der Handwurzel führt insbesondere bei Frakturen mit hohem Dislokationsgrad bzw. hoher Krafteinwirkung relativ oft zu ossären und liga-

OP-JOURNAL 2012; 28: 234-239

(c) Georg Thieme Verlag KG Stuttgart · New York DOI http://dx.doi.org/10.1055/s-0032-1328034 mentären Begleitverletzungen. Von besonderer Bedeutung aufgrund der Häufung ist die Skaphoidfraktur, die Läsion des Discus triangularis und Ruptur des scapholunären Bandes (s. Abb. 1, Kapitel 1). Trotz der Vorteile moderner Implantate, die in vielen Fällen eine anatomische Wiederherstellung und ein gutes radiologisches Ergebnis nach Radiusfraktur ermöglichen, finden sich immer wieder schlechte funktionelle Ergebnisse. Die Ursache hierfür liegt sicherlich zum Großteil an den Begleitverletzungen, die aufgrund der häufig funktionellen Nachbehandlung nicht sicher ausheilen. Dies war sicherlich bei der früher üblichen Ruhigstellung u.U. nicht so offensichtlich, da man spekulieren kann, dass einige - insbesondere Bandverletzungen - dadurch ausgeheilt sind. Auch die besonders fatale Begleitverletzung einer Skaphoidfraktur war dadurch im Falle einer Spätdiagnose zumindest ruhig gestellt. Trotz dieser plausiblen Argumentation bleibt das Ziel der Behandlung die funktionelle Nachbehandlung und das schnelle Erreichen des vollen Bewegungsumfangs. Im Umkehrschluss erfordert dies dann natürlich eine entsprechend genaue Diagnostik und führt nicht selten im Vorfeld der operativen Versorgung zu einer Erweiterung der radiologischen Diagnostik (meist Computertomogramm - CT) bzw. dezidierten intraoperativen Untersuchungsmaßnahmen, um gerade die Stabilität der Handwurzel und des distalen Radioulnargelenks zu beurteilen und dann konsequent die zusätzlichen Verletzungen mitzubehandeln.

\section{Hauptteil}

Die Begleitverletzungen kann man im Wesentlichen in knöcherne und ligamentäre Verletzungen einteilen. Die Behandlungsbedürftigkeit ergibt sich aus dem Schweregrad der Begleitverletzung unter Berücksichtigung der Strategie zur Radiusversorgung, z.B. kann eine unkomplizierte Skaphoidfraktur nach entsprechender Beratung durchaus operativ gleich mitversorgt werden, um eine schneller funktionelle Behandlung zur erreichen. Oftmals erfordert die Versorgung der begleitenden Bandverletzung eine längere Ruhigstellung und kann durchaus dadurch die Technik zur operativen Stabilisierung des Radius beeinflussen oder auch erleichtern, wenn z. B. der erforderliche Zugang von dorsal bei skapholunärer Bandruptur (SL-Band) 
Tab. 1 Klassifikation der karpalen Instabilität.

\begin{tabular}{|c|c|c|c|c|c|}
\hline $\begin{array}{l}\text { Kategorie I } \\
\text { Zeit }\end{array}$ & $\begin{array}{l}\text { Kategorie II } \\
\text { Konstanz }\end{array}$ & $\begin{array}{l}\text { Kategorie III } \\
\text { Ätiologie }\end{array}$ & $\begin{array}{l}\text { Kategorie IV } \\
\text { Lokalisation }\end{array}$ & $\begin{array}{l}\text { Kategorie V } \\
\text { Richtung }\end{array}$ & $\begin{array}{l}\text { Kategorie VI } \\
\text { Muster }\end{array}$ \\
\hline $\begin{array}{l}\text { akut } \\
<1 \text { Woche } \\
\text { subakut } \\
\text { chronisch } \\
>6 \text { Wochen }\end{array}$ & $\begin{array}{l}\text { statisch - nicht } \\
\text { reduzierbar } \\
\text { statisch - reduzierbar } \\
\text { dynamisch } \\
\text { prädynamisch }\end{array}$ & $\begin{array}{l}\text { kongenital } \\
\text { traumatisch } \\
\text { entzündlich } \\
\text { Arthrose } \\
\text { neoplastisch } \\
\text { iatrogen } \\
\text { sonstiges }\end{array}$ & $\begin{array}{l}\text { radiokarpal } \\
\text { prox. interkarpal } \\
\text { mediokarpal } \\
\text { dist. interkarpal } \\
\text { karpometakarpal } \\
\text { knochenspez. }\end{array}$ & $\begin{array}{l}\text { VISI-Rotation } \\
\text { DISI-Rotation } \\
\text { ulnare Translat. } \\
\text { radiale Translat. } \\
\text { palmare Translat. } \\
\text { dorsale Translat. } \\
\text { prox. Translat. } \\
\text { dist. Translat. }\end{array}$ & $\begin{array}{l}\text { karpale Instabilität } \\
\text { dissoziativ (CID) } \\
\text { non-dissoziativ (CIND) } \\
\text { komplex (CIC) } \\
\text { adaptiv (CIA) }\end{array}$ \\
\hline
\end{tabular}

Tab. 2 Mayo-Klassifikation der karpalen Instabilität.

\begin{tabular}{|c|c|c|c|}
\hline I CID & II CIND & III CIC & IV CIA \\
\hline $\begin{array}{l}\text { 1.1. proximale Reihe } \\
\text { a. Skaphoidfraktur } \\
\text { b. SL-Dissoziation } \\
\text { c. LT-Dissoziation } \\
\text { 1.2. distale Reihe } \\
\text { a. axial radial } \\
\text { b. axial ulnar } \\
\text { c. Kombinationen } \\
\text { 1.3. Kombinationen }\end{array}$ & $\begin{array}{l}\text { 2.1. proximale Reihe } \\
\text { a. palmare Bandruptur } \\
\text { b. dorsale Bandruptur } \\
\text { c. Z. n. Fehlheilung } \\
\text { 2.2. distale Reihe } \\
\text { a. ulnopalmare Dislokation } \\
\text { b. radiopalmare Dislokation } \\
\text { c. Kombinationen aus a u. b } \\
\text { d. dorsale Dislokation } \\
\text { 2.3 Kombinationen }\end{array}$ & $\begin{array}{l}\text { a. perilunär mit radiokarpaler } \\
\text { Instabilität } \\
\text { b. perilunär mit axialer Instabilität } \\
\text { c. radiokarpal mit axialer Instabilität } \\
\text { d. SL-Dissoziation mit ulnarer Trans- } \\
\text { lation }\end{array}$ & $\begin{array}{l}\text { a. fehlverheilte Radiusfraktur } \\
\text { b. Madelung-Deformität }\end{array}$ \\
\hline
\end{tabular}
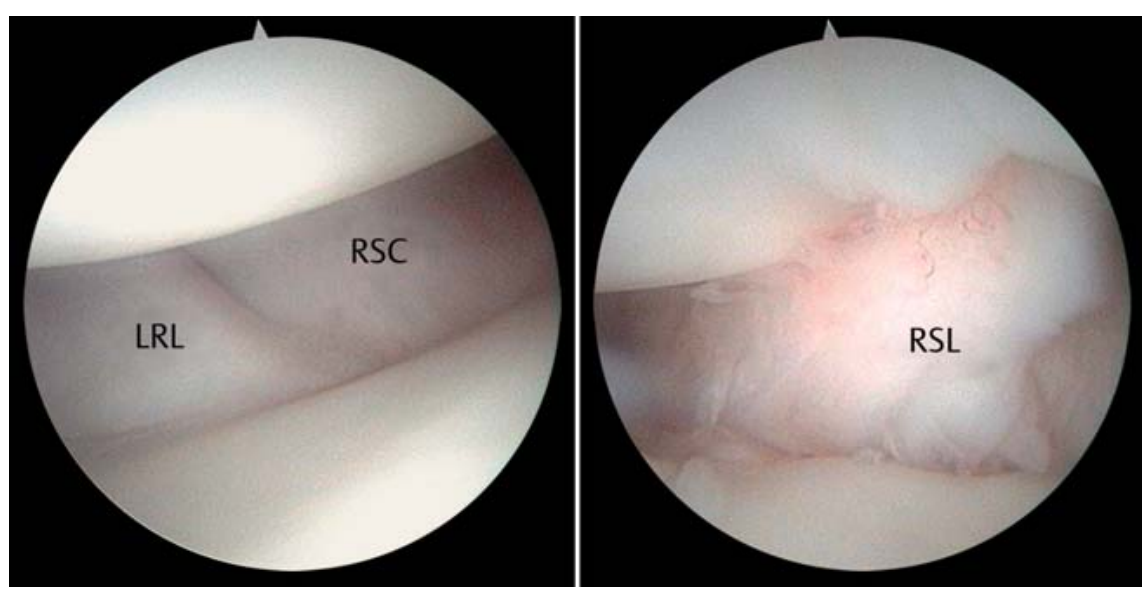

Abb. 1 Arthroskopisches Bild der karpalen Bandstrukturen. RSC: Radioskaphokapitäres Band; LRL: Langes radiolunäres Band; RSL: Radioskapholunäres Band (aus [2]).

dann letztlich auch einen guten Gelenkeinblick ermöglicht [1].

\section{Bandverletzungen}

Das Übersehen einer Verletzung der Bandstrukturen kann zu einem zunehmenden Kollaps der Handwurzel mit erheblicher Schmerzhaftigkeit und Bewegungseinschränkung führen, weshalb dem Erkennen und Wiederherstellen der Strukturen eine erhebliche Bedeutung zukommt. Prinzipiell können bei solchen Bandverletzungen verschiedene Kategorien unterschieden werden wie
Zeit, Konstanz, Ätiologie, Lokalisation und besonders Richtung bzw. Muster. Eine Rotationsfehlstellung findet sich gerade bei den SL-Bandläsionen in der Form einer Dorsalverkippung (DISI: Dorsal Intercalated Segment Instability) und der Palmarverkippung des Lunatums (VISI: Volar Intercalated Segment Instability). Auch bez. des Musters findet sich bei der SL-Bandläsion gehäufter ein Auseinanderweichen von Kahn- und Mondbein als Ausdruck der Dissoziation (CID: Carpal Instability Dissociative) (Tab. 1 und 2). Insbesondere die Bandverbindung von Skaphoid und Lunatum ist hier häufiger betroffen - in eigenen Untersuchungen $8 \%$ mit relevanter Instabilität. Besonders betroffen war der Komplex mit dem Discus triangularis (TFCC) in 25\%, Abrisse des Processus styloideus ulnae und damit ein potenzielles Risiko der Instabilität des DRUG fanden sich in 60\% der Patienten $[3,4,8]$. Der Verdacht auf eine SL-Bandruptur ergibt sich häufig bereits aus der primären Röntgenaufnahme, wenn eine Diastase von Skaphoid und Lunatum festgestellt werden kann. Die Diagnose kann nicht invasiv durch Faustschlussaufnahmen in Ulnarduktion oder mittels Arthrografie bzw. Magnetresonanztomografie (MRT) gesichert werden. Diese Bandrupturen sind jedoch besonders gut im Rahmen einer Handgelenksarthroskopie zu sehen (Abb. 1). Dabei kann deren Stabilität mit dem Tasthaken direkt überprüft und klassifiziert werden, was insbesondere für das skapholunäre Band von erheblicher Bedeutung ist (Tab. 3).

Die Reposition der Handwurzelknochen im Fall der Läsion, temporäre Transfixation (6-8 Wochen) mit entsprechender Ruhigstellung und Refixation der Bandstrukturen ist für die stabile Ausheilung von eminenter Bedeutung (Abb. 2).

Unterbleibt bei Stabilitätsverlust die adäquate Versorgung, muss nach 5-10 Jahren mit einer klinisch relevanten Hand- 


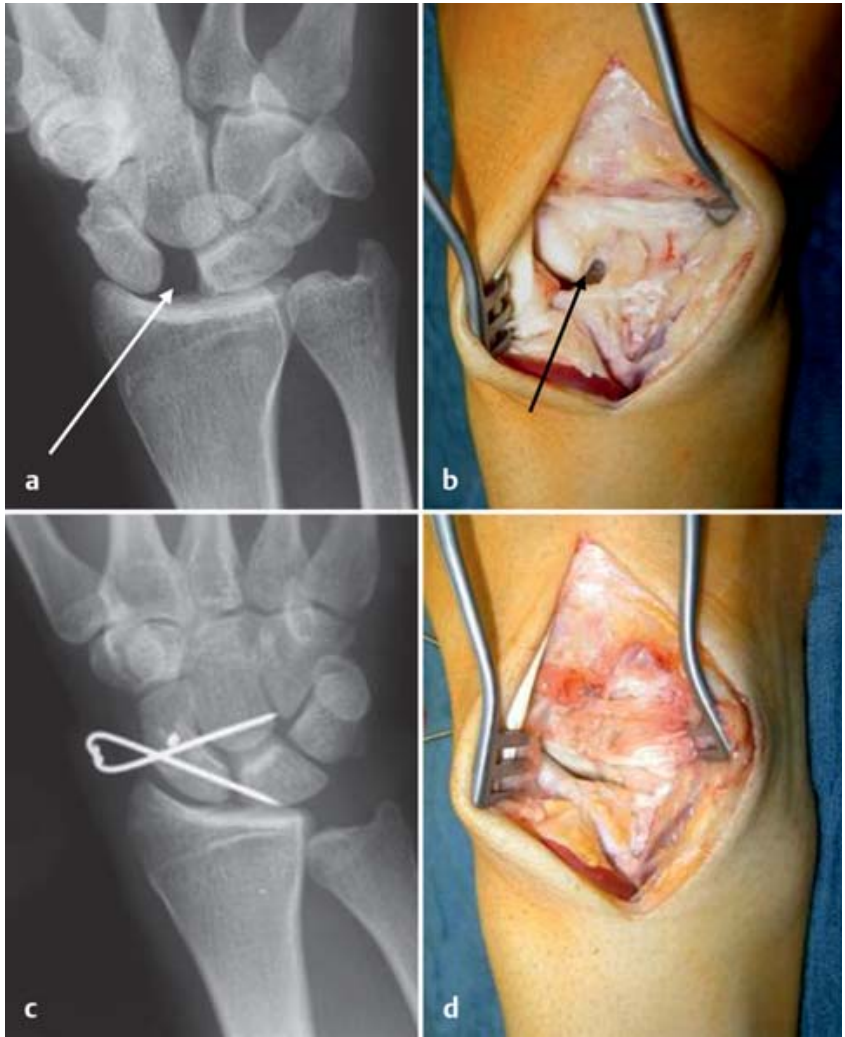

Tab.3 Arthroskopische Klassifikation der Verletzungen des skapholunären und lunotriquetralen Bandes.

\begin{tabular}{ll} 
Schweregrad & arthroskopischer Befund \\
\hline Grad I & $\begin{array}{l}\text { ligamentäre Einblutung (radiokarpal) } \\
\text { keine Inkongruenz mediokarpal }\end{array}$ \\
\hline Grad II & $\begin{array}{l}\text { ligamentäre Einblutung (radiokarpal) } \\
\text { Inkongruenz mediokarpal, Taststab passt nicht interossär }\end{array}$ \\
\hline Grad III & Inkongruenz radiokarpal und mediokarpal \\
& Taststab passt interossär \\
\hline Grad IV & $\begin{array}{l}\text { Inkongruenz radiokarpal und mediokarpal } \\
\text { Instabilität bei Manipulation } \\
\end{array}$ \\
& 2,7 mm Arthroskop passt interossär
\end{tabular}

gelenksarthrose gerechnet werden [7, 9]. Diese wird nach SL-Bandruptur als SLAC-Wrist (Scapho-Lunate-AdvancedCollapse) bezeichnet (Abb. 3).

Besonders betroffen bei der zuvor erwähnten Nachuntersuchung war der Komplex mit dem Discus triangularis (TFCC) in 25\%. Abrisse des Processus styloideus ulnae und damit ein potenzielles Risiko der Instabilität des DRUG fanden sich bei $60 \%$ der Patienten $[3,4$, $6]$.

Wiederum eignet sich besonders die MRT den Weichteilanteil des TFC zu ana-
Abb. 2 Röntgenbilder und intraoperative Bilder einer skapholunären Bandveroperativer Stabilisierung mittels Knochenanker und Anteilen des dorsalen interkarpalen Bandes. a Röntgenbild der Handwurzel mit deutlicher Bandläsion und Dissoziation des Lunatums und Skaphoids. b Intraoperativer Situs mit deutlich sichtbarer Bandläsion. c Röntgenbild nach Reposition und Stabilisierung mittels Knochenanker und 2 Kirschner-Drähten. d Intraoperativer Befund nach Stabilisierung. letzung und deren
Im Zusammenhang mit einer Radiusfraktur und deren operativer Versorgung sollte neben der Prüfung der Stabilität der Handwurzel auch die Festigkeit des DRUG geprüft werden.

Dies erfolgt regelrecht durch Kontrolle in maximaler Pronation und Supination. Zum einen wird dabei eine übermäßige

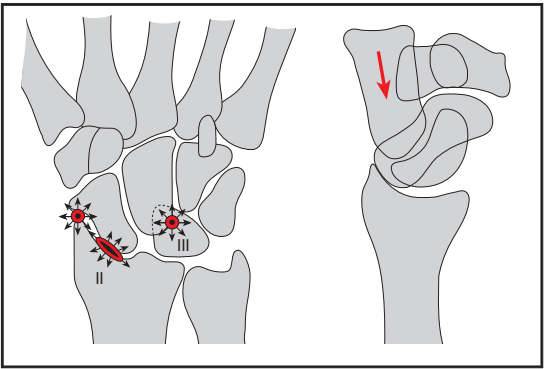

Abb. 3 Schemadarstellung einer SLACWrist. a: Stadien I-III. b: Das Capitatum senkt sich in die Lücke zwischen Skaphoid und Lunatum.

relative Palmardislokation des Radius und zum anderen ein übermäßiges $\mathrm{Ab}$ weichen des Radius nach dorsal überprüft. Findet sich ein solcher Befund, empfiehlt sich die operative Stabilisierung der relevanten Strukturen, entweder durch Naht der beteiligten Bandanteile oder auch die Fixation des abgerissenen Processus styloideus ulnae mittels Knochenanker, Schraube, Zuggurtung oder K-Draht-Fixation (Abb. 7) [6]. Neben dieser relativen Überbeweglichkeit zwischen Radius und Ulna nach palmar oder dorsal muss auch eine übermäßige Translation (z.B. mehr als $5 \mathrm{~mm}$ ) ausgeschlossen werden. Dies gilt insbesondere zum Ausschluss einer Essex-Lopresti-Läsion bei gleichzeitigen Verletzungen des Ellenbogens (z.B. Radiushalsfraktur). Unterbleibt eine exakte Analyse des DRUG, dann muss konsekutiv mit der Entwicklung einer Arthrose gerechnet werden (Abb. 8).

\section{Knöcherne Begleitverletzungen}

Die Untersuchungsergebnisse zeigen, dass bei der distalen Radiusfraktur insbesondere das ulnare Kompartiment und die Strukturen um das DRUG betroffen sind. Hierbei kann die Abrissfraktur des Processus styloideus ulnae als knöcherner Bandabriss gesehen werden und muss wie zuvor erwähnt dann bei Instabilität ausreichend fixiert werden. Davon zu differenzieren ist die distale Ulnafraktur als begleitende knöcherne Verletzung in bis zu 10\% der Fälle. Hier ist zum einen die Fraktur offensichtlich und zum anderen stehen Implantate zur Verfügung, die auch sehr distale Fragmente ausreichend fassen und stabilisieren (Abb.9). Besonderes Augenmerk muss auf die Begleitverletzungen bzw. Frakturen der Handwurzel gerichtet werden (ca. 5\% der Begleitverletzungen). 


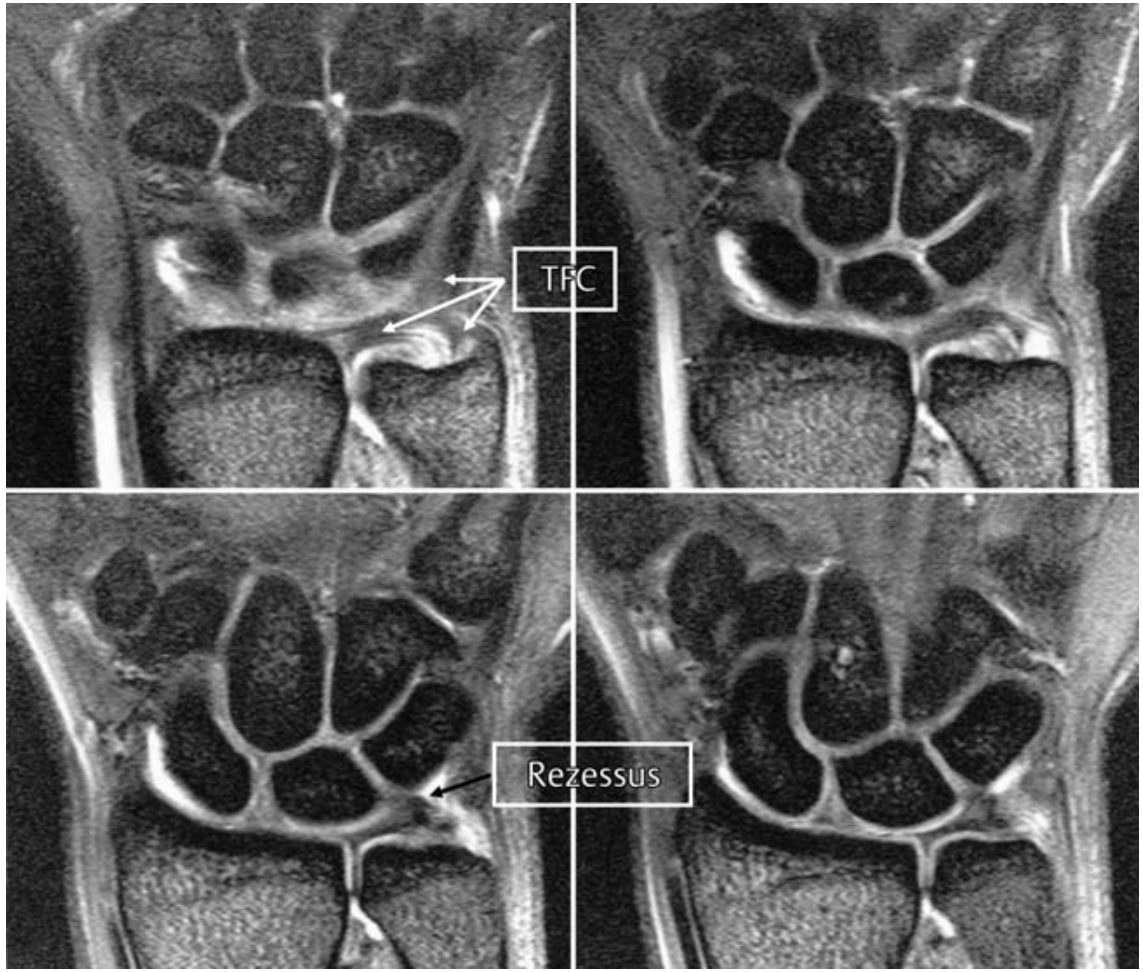

Abb. 4 Kernspintomografisches Bild mit Handwurzelknochen und Discus triangularis (TFC). Die Fasern des TFC, die zum Processus styloideus ulnae bzw. den Handwurzelknochen verlaufen, sind deutlich zu differenzieren. Die kleine palmare Öffnung im TFC (Rezessus) ist ebenfalls zu erkennen und darf nicht mit einer Ruptur verwechselt werden (aus: [2]).

Tab. 4 Klassifikation der traumatischen und degenerativen TFCC- (triangular fibrocartilage complex) Veränderungen.

\begin{tabular}{ll} 
Traumatisch & Degenerativ \\
\hline Klassifikation I: & Klassifikation II: \\
IA: zentrale Perforation des TFC & Stadium 1: TFC-Verschleiß \\
IB: ulnarseitige Avulsion mit/ohne & Stadium 2: TFC-Verschleiß u. Lunatum- u./od. \\
distale Ulnafraktur & Ulna-Chondromalazie \\
IC: distale karpale Avulsion & Stadium 3: TFC-Perforation u. Lunatum- u./od. \\
ID: radialseitige Avulsion mit/ohne & Ulna-Chondromalazie \\
Fraktur der DRUG-Gelenkpfanne & Stadium 4: TFC-Perforation u. Lunatum- u./od. \\
(„Sigmoid Notch“) & Ulna-Chondromalazie u. LT-Bandperforation \\
& Stadium 5: TFC-Perforation u. Lunatum- u./od. \\
& Ulna-Chondromalazie u. LT-Bandperforation u. \\
& ulnokarpale Arthrose
\end{tabular}

Hier steht das Skaphoid ganz im Vordergrund, da es ja in bis zu $80 \%$ der Fälle die häufigste Handwurzelfraktur und Begleitfraktur der Handwurzel bei distaler Radiusfraktur ist $[3,5,10]$.

Diese Begleitverletzungen finden sich gerade beim jungen Patienten (Altersgipfel zwischen 15 und 30 Lebensjahren) und nach Trauma mit hoher Krafteinwirkung. Mit ca. 14\% Anteil bei Handwurzelfrakturen folgt dann das Triquetrum. Alle anderen Frakturen sind generell und in Kombination mit der Radiusfraktur extrem selten (Tab.5). Trotzdem
Das $C T$ ist entscheidend für die Entwicklung einer optimalen Behandlungsstrategie hinsichtlich der Detektion von knöchernen Begleiterscheinungen bei der distalen Radiusfraktur.

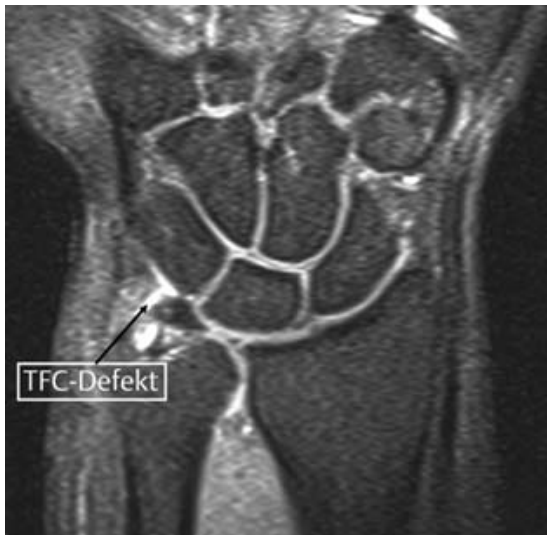

Abb. 5 Kernspintomografische Darstellung einer TFC-Ruptur (aus: [2]).

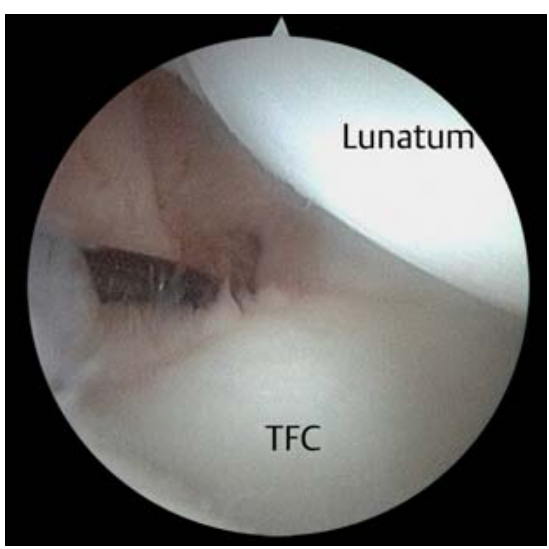

Abb. 6 Arthroskopisches Bild des TFC. Der Tasthaken verschwindet in dem Rezessus (Normalbefund) (aus: [2]).

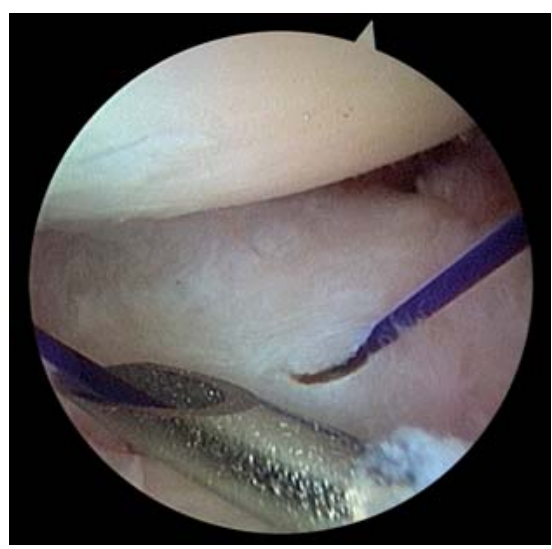

Abb. 7 Arthroskopisches Bild einer ulnarseitigen TFC-Ruptur (1B). Auf der Abbildung sind die Fäden bzw. die Kanüle sichtbar, mit denen die arthroskopische Refixation erfolgte (aus: [2]). 
dorsal

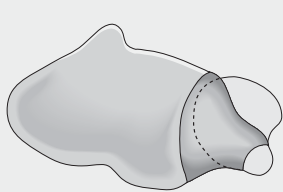

palmar

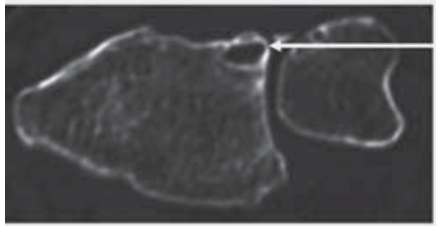

Pronation
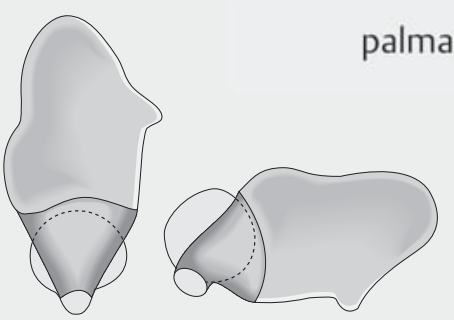

dorsal

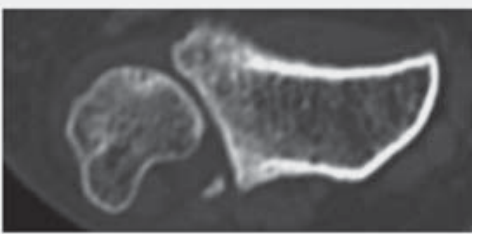

Supination

Abb. 8 Rolle des TFC bei Pronation u. Supination als Stabilisator des distalen Radioulnargelenks. Zusätzlich dargestellt sind computertomografische Bilder bei posttraumatischer Arthrose dieses Gelenks in unterschiedlicher Position. Eine Verschiebung des Radius nach palmar in Relation zur Ulna ist erkennbar als Ausdruck der Instabilität (Pfeil) (aus: [2]).
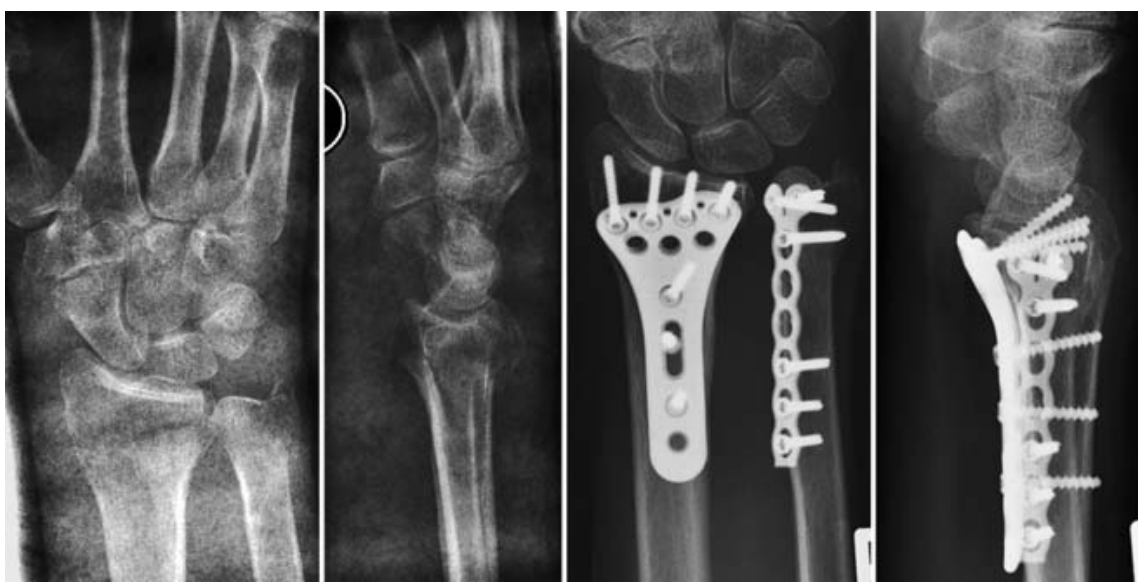

Abb.9 Röntgenbild einer distalen Radiusfraktur mit distaler Ulnafraktur und operativer Versorgung mittels Plattenosteosynthese des Radius und der Elle.
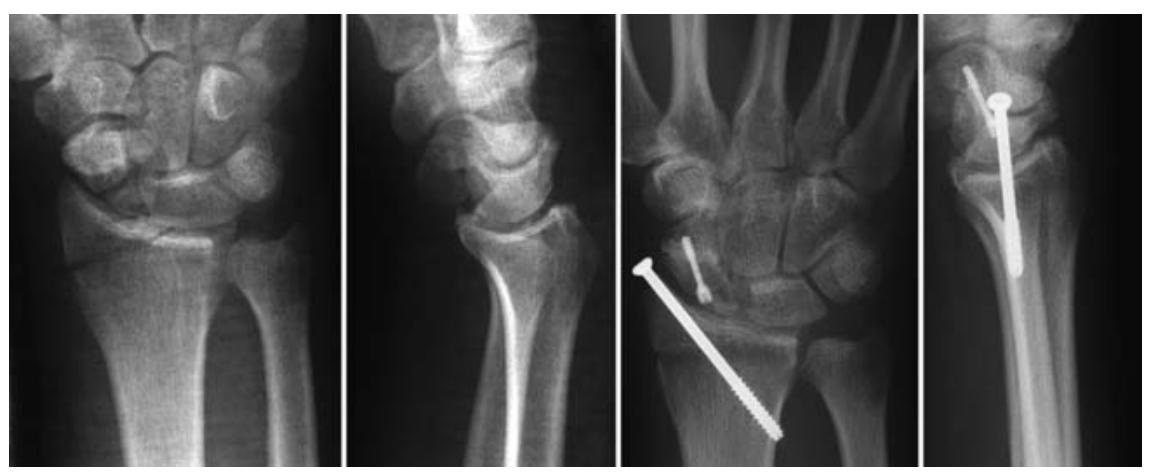

Abb. 10 Röntgenbild einer distalen Radiusfraktur mit Skaphoidfraktur und operativer Versorgung mittels Schraubenosteosynthese des Radius und der Skaphoidfraktur.

Tab.5 Klassifikation der häufigien (Skaphoid, Capitatum, Triquetrum) Handwurzelfrakturen und solcher mit hohen Komplikationen (Lunatum, Hamatum).

Handwurzelknochen

Frakturklassifikation

Skaphoid:

Typ A: stabile Fraktur

- A1 Tuberkulumfraktur

- A2 inkomplette Fraktur der Taille

Typ B: instabile Fraktur

- B1 distal oblique Fraktur

- B2 komplette Fraktur der Taille

- B3 proximale Polfraktur

- B4 perilunäre Luxationsfraktur

Typ C: verzögerte Heilung

Typ D: Pseudarthrose

Lunatum:

- Typ I: Fraktur des palmaren Pols

- Typ II: Knochenabsprengung

- Typ III: Fraktur des dorsalen Pols

- Typ IV: sagittale Lunatumfraktur

- Typ V: transversale Lunatumfraktur

Triquetrum:

- dorsale Avulsionsfraktur

- Korpusfraktur

Hamatum:

- Korpusfraktur

- Gelenkflächenfraktur

- Hamulusfraktur

Capitatum:

- proximale Polfraktur

- Korpusfraktur

- transskaphoidäre, transkapitäre Fraktur

\section{Schlussfolgerung}

Bei Frakturen des distalen Radius müssen unter Berücksichtigung der Unfallursache, des Verletzungsmusters und Dislokationsgrads sowie individueller Voraussetzungen, insbesondere die ligamentären und ossären Strukturen im Bereich des Handgelenks, des distalen Radioulnargelenks und der Handwurzel beachtet werden. Durch die klinische Untersuchung, radiologische Diagnostik mittels korrekter Standardaufnahmen und falls erforderlich Funktionsaufnahmen, CT, MRT, Arthrografie oder Arthroskopie müssen relevante Verletzungen erkannt und mitbehandelt werden.

Die bisherigen Untersuchungsergebnisse zeigen, dass bei guter Rekonstruktion und ausreichender Stabilisierung des Radius die Ergebnisse wesentlich von der optimalen Versorgung der Begleitverletzungen abhängen. 


\section{Literatur}

${ }^{1}$ Fernandez DL, Jupiter JB. Fractures of the distal Radius. A practical Approach to Management. New York, Berlin, Heidelberg: Springer; 1996

2 Frank J, Pralle H, Marzi I. Funktionelle Anatomie und Biomechanik des Handgelenkes und distalen Radioulnargelenkes. OP-JOURNAL 2003; 19: 4-9

${ }^{3}$ Frank J, Pralle H, Lehnert $M$ et al. Begleitverletzungen distaler Radiusfrakturen. Unfallchirurg 2010; 113: 796-803

${ }^{4}$ Geissler WB, Freeland AE, Savoie FH et al. Intracarpal soft-tissue lesions associated with an intra-articular fracture of the distal end of the radius. J Bone Joint Surg [Am] 1996; 78: 357-365

${ }^{5}$ Herbert TJ, Fisher WE. Management of the fractured scaphoid using a new bone screw. J Bone Joint Surg [Br] 1984; 66: 114-123
${ }^{6}$ Lindau T, Adlercreutz C, Aspenberg P. Peripheral tears of the triangular fibrocartilage complex cause distal radioulnar joint instability after distal radial fractures. J Hand Surg Am 2000; 25: 464-468

${ }^{7}$ Rose S, Frank J, Marzi I. Diagnostische und therapeutische Bedeutung der Arthroskopie bei der distalen Radiusfraktur. Zentralbl Chir 1999; 124: 984-992

8 Schneiders W, Amlang M, Rammelt $S$ et al. Häufigkeit der traumatischen und chronischen skapholunären Bandläsion bei distaler Radiusfraktur. Unfallchirurg 2005; 108 : 715-720

${ }^{9}$ Uzdil T, Neumann W, Bauschke A et al. Die palmare winkelstabile Plattenosteosynthese bei distalen Radiusextensionsfrakturen. Akt Traumatol 2001; 4: 141-148

${ }^{10}$ Wolfe SW, Hotchkiss RN, Pederson WC et al. Greens operative Hand Surgery. 6th ed. Philadelphia: Churchill Livingstone; 2011
Prof. Dr. med. Johannes Frank

Stellv. Direktor

\section{Arne Kelm}

Assistenzarzt

Prof. Dr. med. Ingo Marzi

Direktor

Klinik für Unfall-, Hand- und

Wiederherstellungschirurgie

Zentrum der Chirurgie

Klinikum der Johann Wolfgang

Goethe-Universität Frankfurt

Theodor-Stern-Kai 7

60590 Frankfurt am Main

Marzi@trauma.uni-frankfurt.de 